\title{
IMENet: Joint 3D Semantic Scene Completion and 2D Semantic Segmentation through Iterative Mutual Enhancement
}

\author{
Jie Li ${ }^{1,2}$, Laiyan Ding ${ }^{1}$, Rui Huang ${ }^{1,2 *}$ \\ ${ }^{1}$ The Chinese University of Hong Kong, Shenzhen \\ ${ }^{2}$ Shenzhen Institute of Artificial Intelligence and Robotics for Society \\ \{jieli1, laiyanding\}@link.cuhk.edu.cn, ruihuang@cuhk.edu.cn
}

\begin{abstract}
3D semantic scene completion and 2D semantic segmentation are two tightly correlated tasks that are both essential for indoor scene understanding, because they predict the same semantic classes, using positively correlated high-level features. Current methods use 2D features extracted from early-fused RGB-D images for 2D segmentation to improve 3D scene completion. We argue that this sequential scheme does not ensure these two tasks fully benefit each other, and present an Iterative Mutual Enhancement Network (IMENet) to solve them jointly, which interactively refines the two tasks at the late prediction stage. Specifically, two refinement modules are developed under a unified framework for the two tasks. The first is a 2D Deformable Context Pyramid (DCP) module, which receives the projection from the current $3 \mathrm{D}$ predictions to refine the $2 \mathrm{D}$ predictions. In turn, a 3D Deformable Depth Attention (DDA) module is proposed to leverage the reprojected results from 2D predictions to update the coarse 3D predictions. This iterative fusion happens to the stable high-level features of both tasks at a late stage. Extensive experiments on NYU and NYUCAD datasets verify the effectiveness of the proposed iterative late fusion scheme, and our approach outperforms the state of the art on both $3 \mathrm{D}$ semantic scene completion and 2D semantic segmentation.
\end{abstract}

\section{Introduction}

3D Semantic Scene Completion (SSC) has recently drawn increased attention as it aims at simultaneously producing a complete 3D voxel representation of volumetric occupancy and semantic labels from a depth image. Driven by deep Convolutional Neural Networks (CNNs), studies on SSC have achieved significant progress and start to benefit some realworld computer vision and robotics applications, such as indoor navigation, robot grasping, and virtual reality. However, due to the sparsity of 3D data (i.e., most voxels in a 3D scene

\footnotetext{
${ }^{*}$ Corresponding author.
}

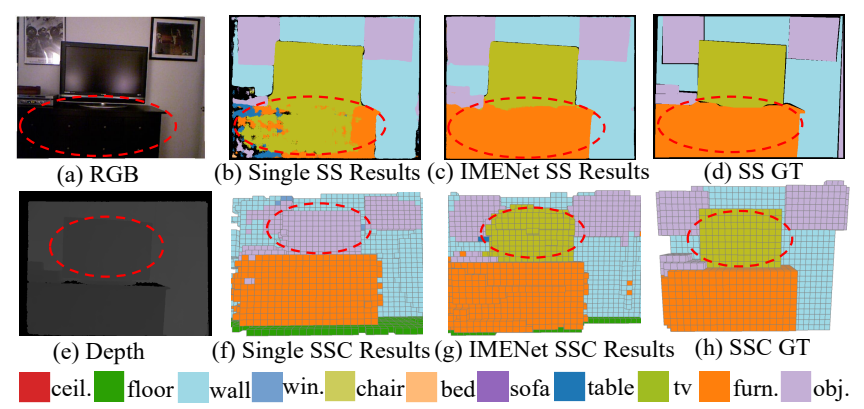

Figure 1: An example of mutual promotion between 3D semantic scene completion (SSC) and 2D semantic segmentation (SS) tasks. The proposed IMENet can improve the accuracy of each other by using the iterative complementary information of different tasks.

are empty), it is a huge challenge for SSC to obtain more accurate results.

On the other hand, 2D Semantic Segmentation (SS) can predict dense class labels from a high-resolution 2D image. Although 2D SS is one dimension lower than 3D SSC, they share some important characteristics that enable them to benefit each other. For example, they produce the same elementwise semantic labels, and both can learn and utilize high-level semantic features under the deep learning framework. Besides, various inexpensive RGB-D imaging devices make the simultaneous acquisition of both data sources very easy. It seems desirable to consider them together so that both get improved. Intuitively, the dense prediction of 2D SS can make up the sparsity of 3D SSC, and the complete shape/layout of the 3D scene can help distinguish inseparable 2D regions.

Several previous studies [Liu et al., 2018; Garbade et al., 2019; Li et al., 2020b] introduced 2D SS into 3D SSC via joint multi-task learning, and improved the SSC accuracy. However, these methods generally perform 2D SS before 3D SSC, and the sequential scheme not only might bring 2D SS errors into 3D SSC, but also provides no runtime feedback from 3D SSC to 2D SS. Besides, the early/shallow fusion of RGB-D multi-modality data in the previous works cannot fully exploit the high-level features of each specific input.

To address the above problems, we propose a novel Iterative Mutual Enhancement Network (IMENet) to realize mutual enhancement between 3D SSC and 2D SS, in an iterative and bi-directional way, on the stable high-level features from 
the late stage of the deep neural network. Our motivation is that geometric information from 3D SSC and high-resolution color/texture features from 2D SS are complementary, and can help the learning of each other. In addition, the iterative interaction between the two tasks allows error-correction based on gradually improved predictions in each task.

Taking the scenario shown in Figure 1 as an example, because of the similar colors/textures of the furniture and $t v$ categories, it is very hard for a 2D SS network to accurately separate the two classes (Figure 1 (b)). On the other hand, since the $t v$ and pictures categories often have similar depth, the existing 3D SSC network may confuse these two classes (Figure 1 (f)). Both failures can be significantly avoided if we leverage the complementary information of the two tasks jointly. Specifically, our proposed IMENet can introduce the distinct geometric shapes of $t v$ and furniture from the SSC branch to SS, and in turn, it can also provide different appearance semantics of $t v$ and pictures from the SS branch to SSC, thus prevent both failures (Figure 1 (c) and (g)).

Additionally, we believe that, even though 2D SS and 3D SSC focus on different channels of the RGB-D input and produce outputs of different dimensions, they share the same semantic labels in the end, and therefore the features learned at the late stages in both tasks are highly correlated. Instead of fusing RGB and D channels early, we adopt a late fusion strategy to fully utilize the correlation of the high-level features from both RGB and D channels. To this end, we designed two task-shared modules named 2D Deformable Context Pyramid (DCP) and 3D Deformable Depth Attention (DDA) for implicit mutual knowledge fusion. In particular, the pixel-wise semantic segmentation and the voxel-wise semantic completion are leveraged to maintain the global-local geometric contexts and accommodate shape and scale variations of different objects through the DCP module. Meanwhile, to probe the inherent property of depth thoroughly, DDA adopts an attention strategy to weigh the depth relationships of different positions after applying the similar DCP structure in 3D. Finally, through iterative interactions between DCP and DDA, our IMENet can explore more refined predictions of both SSC and SS.

In summary, our main contributions are three-fold: 1) We propose a cross-dimensional multi-task iterative mutual enhancement network named IMENet for RGB-D indoor scene understanding. Coarse predictions of task-specific sub-networks can be further refined by properly propagating the complementary information. 2) A late fusion strategy is designed to capture high-level specific features of multimodality input via the 2D Deformable Context Pyramid and 3D Deformable Depth Attention modules. 3) Experimental results on NYU and NYUCAD datasets demonstrate that our IMENet outperforms the state-of-the-art methods.

\section{Related Work}

\subsection{Semantic Segmentation}

The goal of semantic segmentation is to predict class labels for the input raw data, which can be 2D images and 3D volumes. Although 2D dense SS has made great progress due to the rapid development of 2D CNNs [Chen et al., 2017],
3D SS is easier for intelligent agents to understand because of the exact geometries [Qi et al., 2017a; Qi et al., 2017b]. However, it is still challenging to obtain accurate and consistent semantic labels of 3D data because of the inherent sparsity. Therefore, our work will explore interactions between 2D pixel-wise and 3D voxel-wise semantic segmentation to exploit complimentary information for mutual improvements.

\subsection{Semantic Scene Completion}

Semantic Scene Completion methods are divided into two main categories: (1) Single depth-based SSC [Wang et al., 2018b; Wang et al., 2019; Zhang et al., 2019a; Li et al., 2019b] usually reconstructs the semantic complete scenes by solely using the depth as input. In particular, SSCNet [Song et $a l ., 2017]$ is a seminal work that uses an end-to-end dilationbased convolution network to achieve SSC. Although depthbased methods have made considerable progress, the absence of color and texture details always constrains their performance. (2) RGB-D-based SSC can fully use the complementary information from cross-modality inputs to acquire richer features [Chen et al., 2020]. [Li et al., 2019a] first presented a light-weight DDRNet by decomposing the kernel on each dimension. Several works were later proposed to achieve dynamic fusion modes and adaptive kernel size selections [Liu et al., 2020; Li et al., 2020a].

Closer to our work, [Liu et al., 2018] designed a two-stage framework with the SS cascaded by a semantic completion module. Similarly, [Li et al., 2020b] directly conducted 3D semantic completion with 2D SS features as input. In addition, [Garbade et al., 2019] constructed an incomplete 3D semantic tensor from the 2D SS module to formulate the SSC input. However, the sequential arrangement of SS and SS$\mathrm{C}$, as well as the early shallow fusion approaches, have their own limits as discussed in the introduction. Therefore, in this paper we will adopt an iterative and interactive structure between SS and SSC.

\subsection{Multi-task Joint Learning}

Joint learning methods with CNNs have been applied to solve multiple vision tasks simultaneously. For instance, [Zhang et al., 2018b; Zhang et al., 2019b] designed a task-recursive learning (TRL) mechanism, which shares a typical CNN encoder and performs interactions across multiple homogeneous 2D vision tasks at four different scales of the decoder. Different from prior works, our IMENet conducts the iterative interactions between two relatively independent subnetworks, dealing with problems in different dimensions. Besides, the weights of specific sub-networks are updated alternately, which allows every iteration to use the latest and more refined predictions from the other task.

\section{Methodology}

The overall pipeline of our proposed IMENet is shown in Figure 2. The main idea is that the 2D dense segmentation provides semantic priors for 3D semantic completion. Meanwhile, the accurate semantic complete results contribute to producing a correct scheme for image pixel annotations accordingly. The basic strategy is to update the 2D SS branch weights with the 3D SSC weights fixed and vice versa. 


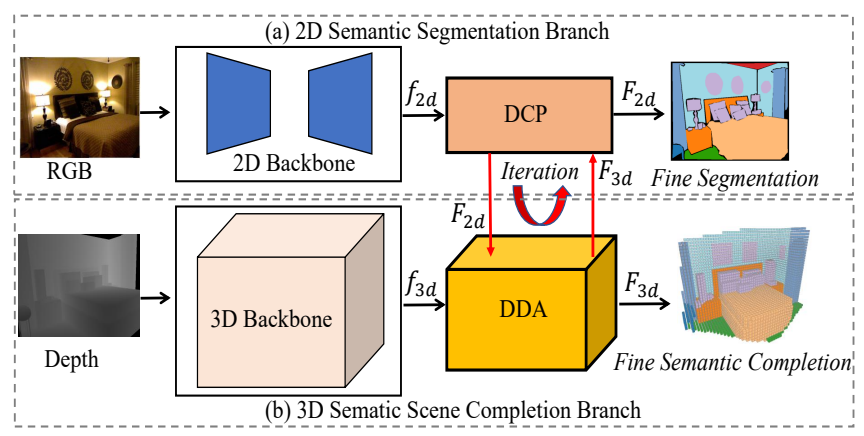

Figure 2: The overall pipeline of our IMENet. It consists of two branches, i.e., (a) 2D semantic segmentation branch and (b) 3D sematic scene completion branch. The red arrows indicate feature interactions between two branches.

\subsection{D Semantic Segmentation Branch}

The semantic segmentation branch aims at acquiring pixelwise semantic predictions of a scene, as shown in Figure 2 (a). Similar to [Liu et al., 2018; Garbade et al., 2019; Li et al., 2020b], we intend to process the SS task in the 2D image space before transferring the segmentation results to the $3 \mathrm{D}$ volume space for time efficiency and higher output resolution. A pre-trained encoder-decoder architecture Seg2DNet [Liu et al., 2018] is applied to realize coarse SS predictions $f_{2 d} \in \mathbb{R}^{12 \times W \times H}$. The encoder is ResNet-101 [He et al., 2016] and the decoder contains a series of dense upsampling convolutions [Wang et al., 2018a]. After the 2D backbone, the coarse result $f_{2 d}$ is sent to the Deformable Context Pyramid (DCP) module to get a further refined semantic prediction map $F_{2 d} \in \mathbb{R}^{1 \times W \times H}$, where $1, W$, and $H$ indicate the channels, width, and height of features.

\subsection{D Semantic Scene Completion Branch}

The goal of the 3D semantic scene completion branch is to map every voxel in the view frustum to one of $K+1$ labels $C=\left\{c_{0}, \ldots, c_{K}\right\}$, where $K(=11$ in this paper $)$ is the number of object classes, and $c_{0}$ represents empty voxels. As shown in Figure 2 (b), the depth input is initially fed into the 3D backbone to generate a 12-channel semantic completion feature map $f_{3 d} \in \mathbb{R}^{12 \times W \times H \times D} . f_{3 d}$ and $F_{2 d}$ are then sent into the Deformable Depth Attention (DDA) module for a better cross-modality feature aggregation. Finally, we can obtain the refined 3D prediction $F_{3 d} \in \mathbb{R}^{1 \times W \times H \times D}$, where $D$ indicates the depth of the features. In addition, The 3D backbone can be replaced by other appealing CNNs.

\subsection{Iterative Mutual Enhancement Framework}

To fully delve into more interactions between two tasks, this paper develops a novel iterative learning framework for closed-loop SSC and SS tasks on indoor scenes (red arrows in Figure 2). The iteration is grounded on the assumption that the better 2D SS results should help achieve better 3D SSC performances and vice versa. The iteration is serialized as a time axis, as shown in Figure 3. Along the time dimension $t$, the two tasks $\left\{S S C_{t}, S S_{t}\right\}$ mutually collaborate to boost the performance of each other. As shown in Figure 2, given an RGB-D image as input, our IMENet first feeds the RGB and $\mathrm{D}$ channels into the $2 \mathrm{D}$ and $3 \mathrm{D}$ backbones respectively, to generate coarse segmentation results $f_{2 d}$ and $f_{3 d}$. $f_{2 d}$ and the 3D feature map $F_{3 d}$ will be fed into DCP for 2D SS refinements. Similarly, $f_{3 d}$ and $F_{2 d}$ are sent to DDA for 3D taskshared fusions. After continuous iterative learning, both the SSC and SS branches gradually improve, and finally, IMENet will output the optimal results $F_{2 d}$ and $F_{3 d}$ respectively.

\subsection{Deformable Context Pyramid}

To incorporate the geometric contextual information and accommodate object shape and scale variations, we introduce a Deformable Context Pyramid (DCP) module in the SS branch of IMENet. In particular, inspired by the outstanding performances of the Atrous Spatial Pyramid Poolings (ASPP) [Chen et al., 2017], we apply a context pyramid structure to aggregate multi-scale contexts and local geometry detail$\mathrm{s}$ to enhance the shape recovery capability of our proposed IMENet. Besides, to improve the geometric transformation modeling capacity, we take advantage of the deformable CNNs [Dai et al., 2017] to keep the shape smooth and consistent.

Figure 4 gives the details of DCP. Given the input $F_{3 d}$ and $f_{2 d}$, we first apply the 3D-2D reprojection layer to project the 3D feature volume $F_{3 d}$ into a 2D feature map $f_{2 d}^{\text {proj. }} \in$ $\mathbb{R}^{1 \times W \times H}$. Then we utilize the one-hot function and deformable context pyramid to transform $f_{2 d}^{\text {proj. }}$ into two new feature maps $f_{2 d}^{1}, f_{2 d}^{2} \in \mathbb{R}^{12 \times W \times H}$, respectively. At last, $f_{2 d}, f_{2 d}^{1}$, and $f_{2 d}^{2}$ are element-wisely added to generate a new feature map $f_{2 d}^{\prime}$. The operation is defined as follows:

$$
\begin{aligned}
f_{2 d}^{\prime} & =D C P_{2 d}\left(f_{2 d}, F_{3 d}\right) \\
& =f_{2 d} \oplus h\left(p\left(F_{3 d}\right)\right) \oplus C R\left[\sum_{i} D_{i}\left(p\left(F_{3 d}\right)\right)\right],
\end{aligned}
$$

where $p, h$ denote the reprojection and one-hot functions respectively, and $\oplus$ means the element-wise sum operation. $C, D_{i}, R$ represent the 2D standard, the $i$ th deformable CNNs and ReLU operations.

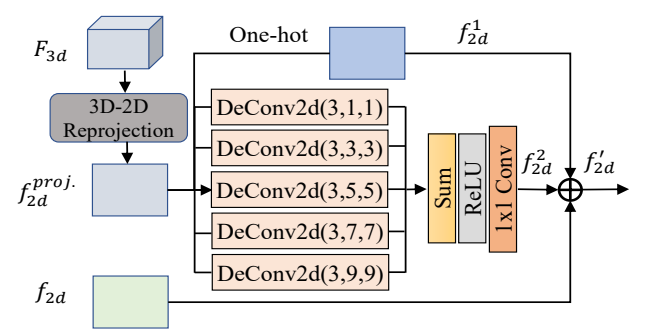

Figure 4: The inner structure of 2D Deformable Context Pyramid.

\subsection{Deformable Depth Attention}

The detailed structure of our Deformable Depth Attention module is illustrated in Figure 5. Due to the similarity between the SSC and SS tasks, we continue to use a similar structure $D C P_{3 d}$ as the first part of our DDA module. The main difference is that we conduct the DCP operation in 3D. 


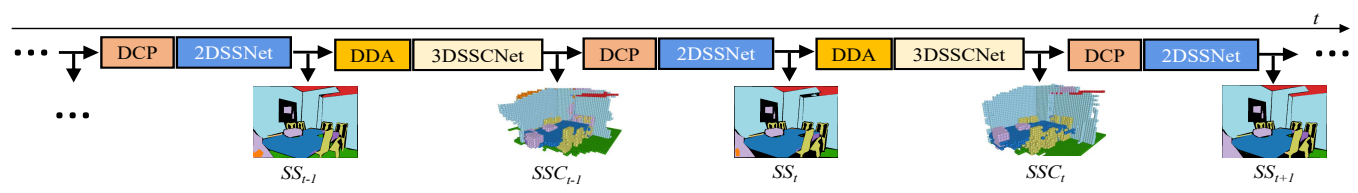

Figure 3: Illustration of our iterative learning. At time slice $t$, the two tasks (i.e., 3D SSC and 2D SS) are progressively refined to form a task state sequence $\left(S S C_{t}, S S_{t}\right)$. 3DSSCNet and 2DSSNet are the 3D SSC branch and 2D SS branch respectively.

In addition, we have empirically observed that voxels belonging to the same semantic category often have a similar or consistent depth, and different objects generally have truncated depths. Therefore, we introduce the depth attention module as the second part of our DDA module to weigh the depth importance of different categories. DDA is formulated as:

$$
\left\{\begin{array}{l}
f_{3 d}^{3}=D C P_{3 d}\left(f_{3 d}, F_{2 d}\right) \\
f_{3 d, j}^{\prime}=\sum_{i=1}^{D}\left(\frac{\exp \left(f_{3 d, i}^{3} \cdot f_{3 d, j}^{3}\right)}{\sum_{i=1}^{D} \exp \left(f_{3 d, i}^{3} \cdot f_{3 d, j}^{3}\right)}\right) f_{3 d, i}^{3}+f_{3 d, j}^{3}
\end{array}\right.
$$

where $f_{3 d}^{3}$ is the output of $D C P_{3 d}$, and $f_{3 d, j}^{\prime}$ is a feature vector in the output feature map $f_{3 d}^{\prime}$ of DDA at the position $j$. Different from the traditional channel attention module [Fu et al., 2019], we directly calculate the depth attention map $X \in \mathbb{R}^{D \times D}$ from the original features $f_{3 d}^{3} \in \mathbb{R}^{12 \times W \times H \times D}$. From Equation (2), it is obvious that the final feature $f_{3 d}^{\prime}$ is a weighted sum of the features of all depth-channels and original features, which models the long-range depth dependencies between feature maps.

\subsection{Loss and Training Protocol}

Loss To train our IMENet, the softmax cross-entropy loss is employed as in [Song et al., 2017]. During training, we remove all empty voxels and do not apply the data balancing scheme in [Song et al., 2017] in our training process.

Training Protocol IMENet is implemented with PyTorch and is trained from scratch. The cross-entropy is minimized by using the standard SGD with a momentum 0.9 and a weight decay 1e-4. The model is trained with 4 RTX 2080Ti GPUs with batch size 4 and initial learning rate 0.005 .

\section{Experiments}

\subsection{Dataset and Metrics}

We evaluate our method on the NYU and NYUCAD datasets. The NYU dataset is a large-scale RGB-D indoor dataset [Silberman et al., 2012], which contains 795/654 (training/testing) aligned pairs of RGB and depth images. Besides, each image is also annotated with a 3D semantic label according to the ground truth completion and segmentation results from [Guo et al., 2015]. However, some manually labeled voxels and their corresponding depths are not well aligned in the NYU dataset. Therefore, we use the NYUCAD dataset [Firman et al., 2016] to tackle these misalignments, in which the depth is projected from the $3 \mathrm{D}$ labeled volume and thus can have higher quality. The voxel-level intersection over union (IoU) between the predicted and ground truth labels is used as the evaluation metric.

\subsection{Comparison with State-of-the-Arts}

Because the 3D backbone used in the 3D SSC branch is flexible and can be replaced by other appealing networks, we conduct experiments on NYUCAD and NYU datasets with two typical networks, i.e., the pioneering SSCNet [Song et al., 2017], and the current state of the art TorchSSC [Chen et al., 2020], to validate the generalization ability of the proposed framework. The results are reported in Table 1 and 2, respectively. In Table 1, we set SSCNet as the 3D backbone. It is obvious that our method significantly outperforms other methods except CCPNet [Zhang et al., 2019a] and TorchSSC in the overall accuracy (mIoU) in the SSC task. The higher accuracy of CCPNet comes from restored details by using full resolution $240 \times 144 \times 240$, while our method just applies to a quarter resolution. Besides, TorchSSC leverages the newly generated 3D sketch priors to guide the SSC task, and since the related data are only released for the NYU dataset, we only use it as our 3D backbone in the experiments on the NYU dataset. Despite that, our IMENet can still obtain competitive results with the relatively early SSCNet as our 3D backbone. Our IMENet achieves 7.5\% mIoU better than the baseline SSCNet, and also $1.5 \%$ higher than the similar SS-based TS3D.

\begin{tabular}{l|lll|l}
\hline Methods & prec. recall IoU & avg. \\
\hline Zheng et al. [Zheng et al., 2013] & 60.1 & 46.7 & 34.6 & - \\
Firman et al. [Firman et al., 2016] & 66.5 & 69.7 & 50.8 & - \\
VVNet [Guo and Tong, 2018] & 86.4 & 92.0 & 80.3 & - \\
DDRNet [Li et al., 2019a]* & 88.7 & 88.5 & 79.4 & 42.8 \\
GRFNet [Liu et al., 2020]* & 87.2 & 91.0 & 80.1 & 45.3 \\
AIC-Net [Li et al., 2020a]* & 88.2 & 90.3 & 80.5 & 45.8 \\
PALNet [Li et al., 2019b] & 87.2 & 91.7 & 80.8 & 46.6 \\
CCPNet [Zhang et al., 2019a] & $\mathbf{9 1 . 3}$ & 92.6 & 82.4 & 53.2 \\
TorchSSC [Chen et al., 2020]* & 90.6 & 92.2 & $\mathbf{8 4 . 2}$ & $\mathbf{5 5 . 2}$ \\
SSCNet [Song et al., 2017] & 75.4 & $\mathbf{9 6 . 3}$ & 73.2 & 40.0 \\
TS3D [Garbade et al., 2019]* & - & - & 76.1 & 46.0 \\
Ours* & $\underline{84.8}$ & 92.3 & $\underline{79.1}$ & $\underline{47.5}$ \\
\hline
\end{tabular}

Table 1: Semantic scene completion results on the NYUCAD dataset with SSCNet as the 3D backbone, and $*$ are RGB-D based methods. Bold numbers represent the best scores and underline marks results that higher than similar methods.

From Table 2 we can see that IMENet maintains the performance advantages and outperforms the 3D backbone TorchSSC by $3.1 \%$ SSC mIoU and $0.8 \%$ SC IoU. Furthermore, IMENet-2DGT, serving as an upper bound for our IMENet, denotes the situation that SS's ground truth is used as the input to the $3 \mathrm{D}$ branch. Our method tends to obtain better performance in some categories with more extreme shape variations, e.g., chair, table, and objects. We argue that this improvement is due to our novel iterative mutual enhancements between the SS-SSC two-branch architecture, enabling 


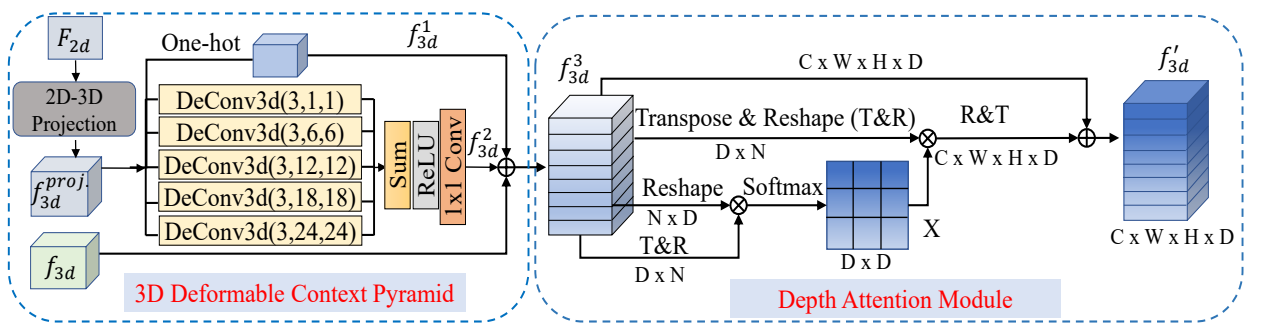

Figure 5: The details of 3D Deformable Depth Attention Module. The kernel, padding and dilation are indicated in the convolutional layers.

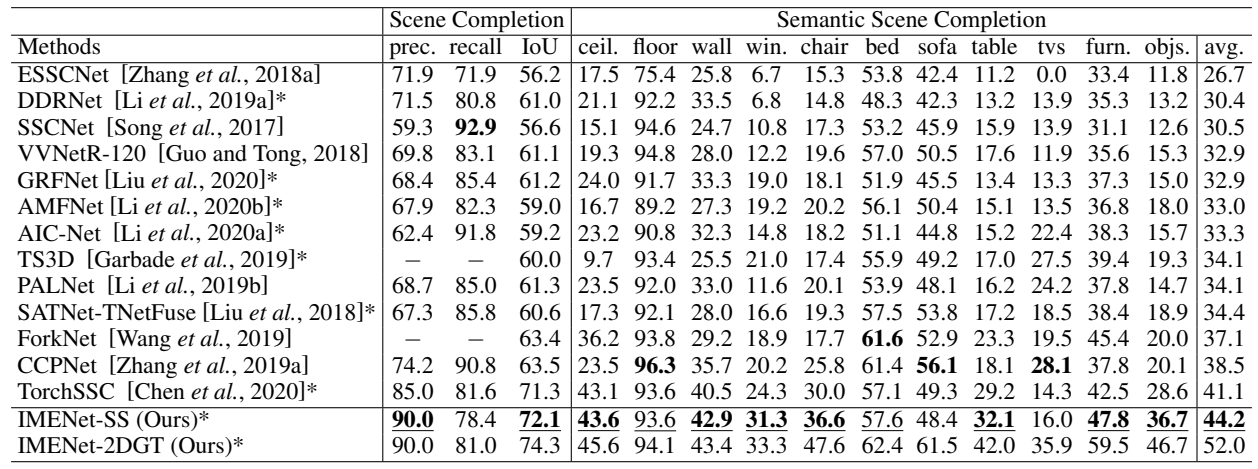

Table 2: Semantic scene completion results on the NYU dataset with TorchSSC as our 3D backbone, and '*' are RGB-D based methods.

SSC to use the 2D semantic prior to the full.

\subsection{Qualitative Analysis}

The visualization of some results on the NYUCAD dataset are shown in Figure 6 to prove the effectiveness of our IMENet qualitatively. Firstly, our proposed method can handle diverse categories with different sizes or various shapes. For example, whether it is a large object window (row 4,6) or a small object $t v$ (row 5), our model can handle it well. The reason lies in that the proposed DCP may adaptively adjust the receptive field voxel-wisely. Besides, for the categories with severe shape variability, such as table in row 4 and objec$t$ in row 3 right rectangle, both SSCNet, and PALNet cannot acquire satisfying shapes. However, our IMENet can model the shape boundary very well, and we attribute this to the complementary color and texture information from the 2D SS branch. Furthermore, our method can also maintain segmentation consistency and perform better on challenging classes than SSCNet and PALNet. The object in row 1 and the right rectangle of row 2, and the window in row 4 demonstrate the superiority of our approach in the segmentation consistency. Within the left red rectangle in row 2 and 3, both SSCNet and PALNet fail to identify the correct category (furniture and object misidentify each other), or they misidentify $t v$ in row 5. But our method still successfully identifies these objects. IMENet can avoid segmentation errors because it has learned the semantic priors from the 2D SS branch.

\subsection{Ablation Study}

Different Modules in the Framework We first conduct ablation studies on different modules in our IMENet, as shown in Table 3. The baseline (model A) is set to learn SSC and

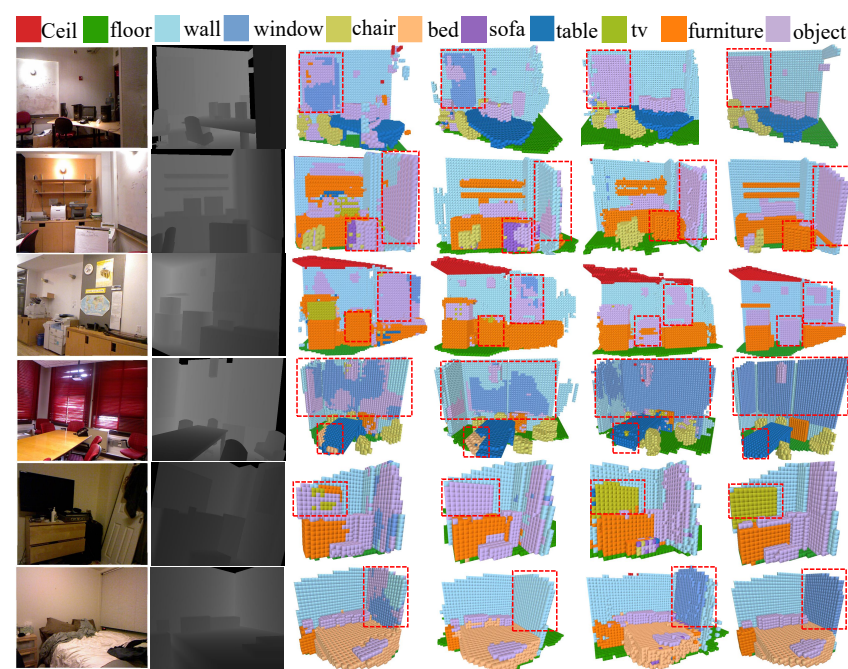

(a) RGB

(b) Depth

(c) SSCNet

(d) PALNet

(e) Ours

(f) GT

Figure 6: Qualitative results on the NYUCAD dataset. From left to right: (a-b) Input RGB-D images, (c) results obtained by SSCNet [Song et al., 2017], (d) results generated by PALNet [Li et al., 2019a], (e) the proposed approach, and (f) ground truth.

\begin{tabular}{c|ccc|c|cc}
\hline Model & IL & DCP & DDA & SS & SSC & SC \\
\hline A & $\boldsymbol{x}$ & $\boldsymbol{x}$ & $\boldsymbol{x}$ & 46.8 & 40.3 & 72.3 \\
B & $\checkmark$ & $\boldsymbol{x}$ & $\boldsymbol{x}$ & 48.1 & 42.5 & 74.3 \\
C & $\checkmark$ & $\checkmark$ & $\boldsymbol{x}$ & 62.2 & 42.8 & 75.5 \\
D & $\checkmark$ & $\boldsymbol{x}$ & $\checkmark$ & 47.4 & 44.8 & 77.7 \\
E & $\boldsymbol{}$ & $\boldsymbol{\checkmark}$ & $\checkmark$ & $\mathbf{6 5 . 6}$ & $\mathbf{4 7 . 5}$ & $\mathbf{7 9 . 1}$ \\
\hline
\end{tabular}

Table 3: Ablation study on IL/DCP/DDA on the NYUCAD dataset. 
SS separately without iterative learning (IL). Model A only gets mIoU of $46.8 \%$ on SS and $40.3 \%$ on SSC. Model B with IL means that we perform iterative learning several times, but there is no feature interaction between the two branches. The SS performance of model B is slightly improved to $48.1 \%$, and both SC and SSC are about 2\% higher than model A. Moreover, Model C and D are used to verify the effectiveness of the proposed DCP and DDA modules. After the interactive features are applied via DCP, SS is greatly improved to $62.2 \%$ by a large margin (14.1\%). Similarly, improvements of $2.3 \%$ and $3.4 \%$ on SSC and SC are further obtained through DDA. Furthermore, with both DCP and DDA added to the overall pipeline, our IMENet achieves the best results on both tasks, which are presented in bold numbers.

In addition, the 2D SS accuracy comparisons are shown in Table 4. We select Seg2DNet-RGB [Liu et al., 2018] as our $2 \mathrm{D}$ backbone of all the experiments in this paper because it released the pre-trained model. When 3D SSC features with geometric priors flow into the $2 \mathrm{D}$ branch via the DCP module, 2D SS predictions are significantly improved compared with RGB-D as input directly (denoted by Seg2DNetRGBD). Deeplab v3+ is pretrained on ADE-20k [Zhou et al., 2017] and finetuned on NYU with a CRF applied on the output. Although the average IoU of our method is slightly lower than Deeplab v3+, it is much higher than other methods without any post-processing. Furthermore, IMENet can achieve the best results in some challenging categories (e.g., chair).

\begin{tabular}{|c|c|c|c|c|}
\hline Methods & $\begin{array}{l}\text { Sele } \\
\text { wall }\end{array}$ & $\begin{array}{l}\text { ted } 3 \\
\text { chair }\end{array}$ & $\begin{array}{l}\text { lasses } \\
\text { objs. }\end{array}$ & $\begin{array}{l}11 \text { classes } \\
\text { avg. IoU }\end{array}$ \\
\hline Deeplab v2 [Garbade et al., 2019] & 76.6 & 58.5 & 54.7 & 61.5 \\
\hline Deeplab v3+ [Garbade et al., 2019] & 82.8 & 65.8 & 62.9 & 69.3 \\
\hline Seg2DNet [Liu et al., 2018] & 54.3 & 42.8 & 39.8 & 46.8 \\
\hline Seg2DNet-RGBD [Liu et al., 2018] & 58.8 & 44.7 & 42.3 & 48.3 \\
\hline IMENet-SSC (Ours) & 86.0 & 77.0 & 76.1 & 65.6 \\
\hline IMENet-3DGT (Ours) & 86.6 & 90.6 & 98.6 & 80.3 \\
\hline
\end{tabular}

Table 4: 2D Semantic segmentation results on the NYU dataset. In both cases of the upper part, the pre-trained models on other datasets and a CRF are used. Underlines are higher than backbone methods.

Impacts of DCP and DDA To further validate the effectiveness of DCP and DDA, the comparison results are shown in Figure 7 and Figure 8, respectively. We can observe that DCP can successfully identify different categories, even if their colors/textures are very similar, as shown in red squares. Take the first row in Figure 7 as an example, because the colors/textures of chair and floor are similar, the IMENet (w/o DCP) will easily mislabel floor as chair, while IMENet (w DCP) can rectify this error. We attribute this to the DCP's ability to effectively integrate shape/depth geometries introduced from the 3D branch. Similarly, DDA can scrutinize more discriminative contexts even though they have similar depths, and we owe it to the advantage that DDA can adequately fuse the colors/textures obtained from the 2D branch.

Analysis of Iterative Numbers Experiments are conducted to analyze the impact of different iterative numbers, and the accuracies of both SSC and SS tasks are shown in Figure 9. It can be observed that the results first increase with more iterations, then they get stable around 3 and 4 . Fur-

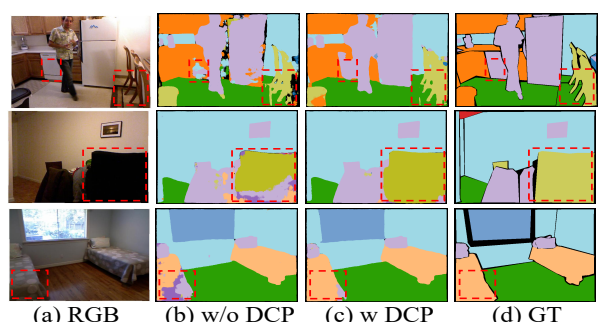

Figure 7: Qualitative comparison between our approach w/o and w/ DCP on the NYU validation set.

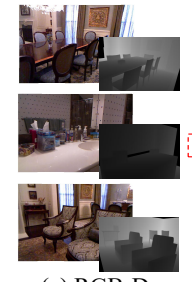

(a) RGB-D

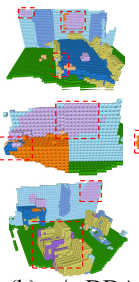

(b) w/o DDA (c) W DDA

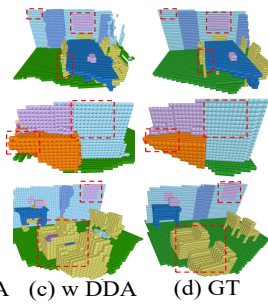

(d) GT
Figure 8: Qualitative comparison between our approach w/o and w/ DDA on the NYUCAD validation set.

ther increasing the number of iterations degrades the performance. This phenomenon is consistent with the observation of [Zhang et al., 2019b]. The network convergence may explain this phenomenon. In general, using more iterations can improve the performance to some extent. But more iterations also require more training for the network to converge.

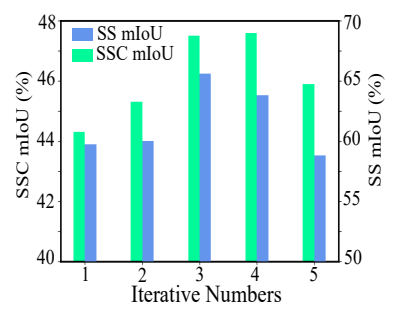

Figure 9: Accuracies of SSC and SS with different iterative times.

\section{Conclusion}

This paper proposes an iterative mutual enhancement network named IMENet to further exploit bi-directional fusions between semantic completion and segmentation tasks via two task-shared refined modules, i.e., Deformable Context Pyramid and Deformable Depth Attention. By leveraging the late fusion method, our IMENet can fully explore specific highlevel features of multi-modality input. Finally, elaborate experiments on both NYU and NYUCAD datasets show that our proposed IMENet achieves state-of-the-art results on both semantic scene completion and semantic segmentation tasks.

\section{Acknowledgments}

This work was supported in part by Shenzhen Natural Science Foundation under Grant JCYJ20190813170601651, and in part by funding from Shenzhen Institute of Artificial Intelligence and Robotics for Society. 


\section{References}

[Chen et al., 2017] Liang-Chieh Chen, George Papandreou, Iasonas Kokkinos, Kevin Murphy, and Alan L Yuille. Deeplab: Semantic image segmentation with deep convolutional nets, atrous convolution, and fully connected crfs. TPAMI, 40(4):834-848, 2017.

[Chen et al., 2020] Xiaokang Chen, Kwan-Yee Lin, Chen Qian, Gang Zeng, and Hongsheng Li. 3D sketch-aware semantic scene completion via semi-supervised structure prior. In CVPR, pages 4193-4202, 2020.

[Dai et al., 2017] Jifeng Dai, Haozhi Qi, Yuwen Xiong, Yi Li, Guodong Zhang, Han $\mathrm{Hu}$, and Yichen Wei. Deformable convolutional networks. In $I C C V$, pages 764$773,2017$.

[Firman et al., 2016] Michael Firman, Oisin Mac Aodha, Simon Julier, and Gabriel J Brostow. Structured prediction of unobserved voxels from a single depth image. In $C V P R$, pages 5431-5440, 2016.

[Fu et al., 2019] Jun Fu, Jing Liu, Haijie Tian, Yong Li, Yongjun Bao, Zhiwei Fang, and Hanqing Lu. Dual attention network for scene segmentation. In CVPR, pages 31463154, 2019.

[Garbade et al., 2019] Martin Garbade, Yueh-Tung Chen, Johann Sawatzky, and Juergen Gall. Two stream 3D semantic scene completion. In CVPRW, pages 416-425, 2019.

[Guo and Tong, 2018] Yu-Xiao Guo and Xin Tong. Viewvolume network for semantic scene completion from a single depth image. arXiv preprint arXiv:1806.05361, 2018.

[Guo et al., 2015] Ruiqi Guo, Chuhang Zou, and Derek Hoiem. Predicting complete 3D models of indoor scenes. arXiv preprint arXiv:1504.02437, 2015.

[He et al., 2016] Kaiming He, Xiangyu Zhang, Shaoqing Ren, and Jian Sun. Deep residual learning for image recognition. In CVPR, pages 770-778, 2016.

[Li et al., 2019a] Jie Li, Yu Liu, Dong Gong, Qinfeng Shi, Xia Yuan, Chunxia Zhao, and Ian Reid. RGBD based dimensional decomposition residual network for 3D semantic scene completion. In CVPR, pages 7693-7702, 2019.

[Li et al., 2019b] Jie Li, Yu Liu, Xia Yuan, Chunxia Zhao, Roland Siegwart, Ian Reid, and Cesar Cadena. Depth based semantic scene completion with position importance aware loss. In RAL, 5(1):219-226, 2019.

[Li et al., 2020a] Jie Li, Kai Han, Peng Wang, Yu Liu, and Xia Yuan. Anisotropic convolutional networks for 3D semantic scene completion. In CVPR, pages 3351-3359, 2020 .

[Li et al., 2020b] Siqi Li, Changqing Zou, Yipeng Li, Xibin Zhao, and Yue Gao. Attention-based multi-modal fusion network for semantic scene completion. In $A A A I$, pages 11402-11409, 2020.

[Liu et al., 2018] Shice Liu, Yu Hu, Yiming Zeng, Qiankun Tang, Beibei Jin, Yinhe Han, and Xiaowei Li. See and think: Disentangling semantic scene completion. In NIPS, pages 263-274, 2018.
[Liu et al., 2020] Yu Liu, Jie Li, Qingsen Yan, Xia Yuan, Chunxia Zhao, Ian Reid, and Cesar Cadena. 3D gated recurrent fusion for semantic scene completion. arXiv preprint arXiv:2002.07269, 2020.

[Qi et al., 2017a] Charles R Qi, Hao Su, Kaichun Mo, and Leonidas J Guibas. PointNet: Deep learning on point sets for $3 \mathrm{D}$ classification and segmentation. In $C V P R$, pages 652-660, 2017.

[Qi et al., 2017b] Charles Ruizhongtai Qi, Li Yi, Hao Su, and Leonidas J Guibas. PointNet++: Deep hierarchical feature learning on point sets in a metric space. In NIPS, pages 5099-5108, 2017.

[Silberman et al., 2012] Nathan Silberman, Derek Hoiem, Pushmeet Kohli, and Rob Fergus. Indoor segmentation and support inference from RGBD images. In ECCV pages 746-760. Springer, 2012.

[Song et al., 2017] Shuran Song, Fisher Yu, Andy Zeng, Angel X Chang, Manolis Savva, and Thomas Funkhouser. Semantic scene completion from a single depth image. In CVPR, pages 1746-1754, 2017.

[Wang et al., 2018a] Panqu Wang, Pengfei Chen, Ye Yuan, Ding Liu, Zehua Huang, Xiaodi Hou, and Garrison Cottrell. Understanding convolution for semantic segmentation. In WACV, pages 1451-1460. IEEE, 2018.

[Wang et al., 2018b] Yida Wang, David Joseph Tan, Nassir Navab, and Federico Tombari. Adversarial semantic scene completion from a single depth image. In $3 D \mathrm{~V}$, pages 426 434, 2018.

[Wang et al., 2019] Yida Wang, David Joseph Tan, Nassir Navab, and Federico Tombari. ForkNet: Multi-branch volumetric semantic completion from a single depth image. In ICCV, pages 8608-8617, 2019.

[Zhang et al., 2018a] Jiahui Zhang, Hao Zhao, Anbang Yao, Yurong Chen, Li Zhang, and Hongen Liao. Efficient semantic scene completion network with spatial group convolution. In $E C C V$, pages 733-749, 2018.

[Zhang et al., 2018b] Zhenyu Zhang, Zhen Cui, Chunyan X$\mathrm{u}$, Zequn Jie, Xiang Li, and Jian Yang. Joint task-recursive learning for semantic segmentation and depth estimation. In $E C C V$, pages 235-251, 2018.

[Zhang et al., 2019a] Pingping Zhang, Wei Liu, Yinjie Lei, Huchuan Lu, and Xiaoyun Yang. Cascaded context pyramid for full-resolution 3D semantic scene completion. In ICCV, pages 7801-7810, October 2019.

[Zhang et al., 2019b] Zhenyu Zhang, Zhen Cui, Chunyan X$\mathrm{u}$, Zequn Jie, Xiang Li, and Jian Yang. Joint task-recursive learning for RGB-D scene understanding. TPAMI, 2019.

[Zheng et al., 2013] Bo Zheng, Yibiao Zhao, Joey C Yu, Katsushi Ikeuchi, and Song-Chun Zhu. Beyond point clouds: Scene understanding by reasoning geometry and physics. In $C V P R$, pages 3127-3134, 2013.

[Zhou et al., 2017] Bolei Zhou, Hang Zhao, Xavier Puig, Sanja Fidler, Adela Barriuso, and Antonio Torralba. Scene parsing through ade20k dataset. In CVPR, July 2017. 